\title{
Coupled-oscillator system with two stable phase-shift intervals
}

\author{
Franco Ramírez, Almudena Suárez, Sergio Sancho \\ University of Cantabria, Santander, Spain
}

\begin{abstract}
In this work, a coupled-oscillator system with coupling networks containing discrete transmissions lines, made up of inductors and varactors, is presented. Under weak coupling conditions, it is possible to obtain two stable phase-shift intervals, $-90^{\circ}$ to $90^{\circ}$ and $90^{\circ}$ to $270^{\circ}$, by using two different values of the varactor bias voltage. For each of these two bias voltages, the inter-stage phase shift is varied with the tuning voltage of the outermost oscillators, so the use of the discrete lines provides a simple mechanism to increase the phase-shifting capabilities. The two bias voltages of the line varactor diodes are selected so as to obtain optimum operation conditions in each phase interval, with minimum frequency deviation, maximum and unambiguous tuning parameter excursion and a flat phase-noise response versus the imposed phase shift. The impact of the novel type of coupling network on the stability properties is investigated with an explicit semi-analytical formulation. Agreement has been obtained with measured results.
\end{abstract}

Keywords-Coupled oscillators; stability; phase noise; discrete transmission lines

\section{INTRODUCTION}

Coupled oscillator systems can be applied for spatial power combination and beam steering of phased-array antennas [1-3]. As demonstrated in [1], the phase shift $\phi$ between the oscillator elements can be varied by detuning the outermost oscillators only, which, in comparison with traditional methodologies, avoids the need for phase shifters. The circuit-level analysis of the whole system is computationally expensive and fails to converge in some cases [4]. To cope with this problem, a reduced-order semianalytical formulation has been proposed in [5-6]. This relies on the use of a numerical admittance-type model for the oscillator elements, extracted from a harmonic balance (HB) simulation of the individual oscillator, in free-running regime. The effect of the nearest-neighbor coupling is analyzed considering these models and the admittance matrix $\left[Y_{c}\right]$ that describes the coupling networks, usually made up of transmission lines bounded by resistors. Under weak coupling operation, the explicit formulation in [6] has enabled the derivation of mathematical conditions for an optimum system design. These conditions ensure a minimum oscillation frequency deviation $(\Delta \omega)$ and a flat phase noise response when changing the tuning voltages $\left(\Delta \eta_{1}, \Delta \eta_{2}\right)$ of the outermost oscillators across the stable phase shift interval, which can be either $\left(-90^{\circ}, 90^{\circ}\right)$ or $\left(90^{\circ}, 270^{\circ}\right)$ [6-7]. Under these optimum operation conditions, maximum and unambiguous excursions of $\left(\Delta \eta_{1}, \Delta \eta_{2}\right)$ are also obtained, such that a same tuning value cannot give rise to more than one inter-stage phase shift. For a given free-running oscillator design, the stable phase shift interval and optimum-operation conditions mainly depend on the length of the transmission line in the coupling networks. Taking this dependence into account, the possibility to use a discrete transmission line composed of inductors and varactors is investigated here. The aim is to obtain two different values of the line varactor bias voltage $\left(V_{\text {bias }}\right)$, each enabling an optimum design at one of the two possible stable phase-shift intervals: $\left(-90^{\circ}, 90^{\circ}\right)$ and $\left(90^{\circ}, 270^{\circ}\right)$. The coupled-oscillator system will be analyzed with an explicit semi-analytical formulation, depending on $V_{\text {bias }}$, which will be validated through comparison with costly HB simulations. The possible impact of the new type of coupling network on the size of the stable intervals will be investigated with an extended stability formulation. The new method has been applied to a coupled oscillator system at 3.9 $\mathrm{GHz}$ with good experimental results.
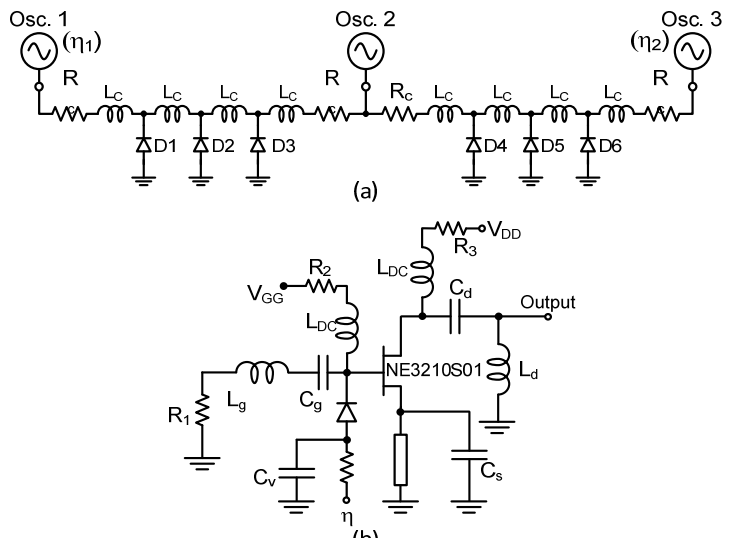

(b)

Fig. 1 Coupled-oscillator system with coupling networks based on inductorvaractor transmission lines. (a) Coupled system. (b) Oscillator design.

\section{COUPLED-OSCILLATOR SYSTEM WITH DISCRETE TRANSMISSION LINES}

Let a discrete transmission line composed of $n$ sections of inductance $L$ and varactor diodes $C(\mathrm{v})$ be considered. In the limit of a nearly continuous line, the delay per section can be approached $\Delta \tau(v)=\sqrt{L C(v)} \quad[8]$, and, in small-signal conditions, this delay should decrease with the reverse bias voltage $V_{\text {bias }}$. The coupling network considered here is composed by a short discrete transmission line, bounded by high value resistors $R$, which should ensure a weak-coupling operation of the oscillator system [Fig. 1(a)]. The coupledsystem analysis relies on the application of Kirchoff's laws to the nodes at which the $N$ oscillators, described with 
admittance-type models, are coupled to the system. For this analysis, the coupling networks (including the $L$-varactor stages and bounding resistors) will be realistically described with their admittance matrix, depending on $V_{\text {bias }}$. For a configuration of the type shown in Fig. 1(a), the biasdependent admittance matrix will have the form:

$$
\left[Y_{c}\left(V_{\text {bias }}\right)\right]=\left[\begin{array}{cc}
Y_{e}\left(V_{\text {bias }}\right) & Y_{n b}\left(V_{\text {bias }}\right) \\
Y_{n b}\left(V_{\text {bias }}\right) & Y_{e}\left(V_{\text {bias }}\right)
\end{array}\right]
$$

The above matrix can be easily calculated through an independent simulation of the coupling network. Next the coupled-oscillator system will be formulated. As derived in [6,9], with a system of only $N=3$ oscillator elements it is possible to predict the steady-state behavior of a system with an arbitrary number $N$, in terms of frequency and amplitude deviations and parameter-values $\left(\Delta \eta_{1}, \Delta \eta_{2}\right)$ required for each phase shift $\phi$. Assuming a constant inter-stage phase shift $\phi$, and taking into account the coupling matrix in (1), the 3-element system is described as:

$$
\begin{aligned}
& {\left[Y_{V} \Delta V_{1}+Y_{\omega} \Delta \omega+Y_{\eta} \Delta \eta_{1}\right] V_{0}=-Y_{e}\left(V_{\text {bias }}\right) V_{0}-Y_{n b}\left(V_{\text {bias }}\right) V_{0} e^{j \phi}} \\
& {\left[Y_{V} \Delta V_{2}+Y_{\omega} \Delta \omega\right] V_{0}=-Y_{n b} V_{0} e^{-j \phi}-2 Y_{e}\left(V_{\text {bias }}\right) V_{0}-Y_{n b}\left(V_{\text {bias }}\right) V_{0} e^{j \phi}} \\
& {\left[Y_{V} \Delta V_{3}+Y_{\omega} \Delta \omega+Y_{\eta} \Delta \eta_{2}\right] V_{0}=-Y_{n b}\left(V_{\text {bias }}\right) V_{0} e^{-j \phi}-Y_{e}\left(V_{\text {bias }}\right) V_{0}}
\end{aligned}
$$

where $V_{0}$ is the free-running voltage, $\Delta V_{i}$, with $i=1,2,3$, are the amplitude deviations and $Y_{V}, Y_{\omega}, Y_{\eta}$ are the derivatives of the oscillator admittance function [6] with respect to the fundamental-frequency voltage amplitude, frequency and oscillator tuning voltage. Second order terms: $\Delta V_{i}^{2}, \Delta V_{i} \Delta \omega, \Delta V_{i} \Delta \eta$ have been neglected in (2), which is enabled by the weak-coupling operation. With the $L$-varactor coupling networks, a stable phase shift interval $\left(-90^{\circ}, 90^{\circ}\right)$ with optimum behavior (as defined in the introduction) will be obtained when the two following conditions [6] are fulfilled at a particular bias voltage $V_{\text {bias } 1}$ :

$$
\begin{aligned}
& \sin \left[\operatorname{ang}\left(Y_{n b}\left(V_{\text {bias } 1}\right)\right)-\operatorname{ang}\left(Y_{V}\right)\right]=\sin \left[\alpha_{v, n b}\left(V_{\text {bias } 1}\right)\right]=0 \\
& \cos \left[\operatorname{ang}\left(Y_{n b}\left(V_{\text {bias } 1}\right)\right)-\operatorname{ang}\left(Y_{V}\right)\right]=\cos \left[\alpha_{v, n b}\left(V_{\text {bias } 1}\right)\right]=-1
\end{aligned}
$$

In turn, a stable phase shift interval $\left(90^{\circ}, 270^{\circ}\right)$ with optimum behavior [6] will be obtained for:

$$
\begin{aligned}
& \sin \left[\alpha_{v, n b}\left(V_{\text {bias } 2}\right)\right]=0 \\
& \cos \left[\alpha_{v, n b}\left(V_{\text {bias } 2}\right)\right]=1
\end{aligned}
$$

For a given oscillator design, with a particular angle of the amplitude derivative $Y_{V}$, the phase values required are $\operatorname{ang}\left(Y_{n b}\right)=(2 k+1) \pi+\operatorname{ang}\left(Y_{V}\right)$, where $k$ is an integer, for the stable interval $\left(-90^{\circ}, 90^{\circ}\right)$, and $\operatorname{ang}\left(Y_{n b}\right)=2 k \pi+\operatorname{ang}\left(Y_{V}\right)$, for the stable interval $\left(90^{\circ}, 270^{\circ}\right)$.

Here a coupled system of three oscillator elements $(N=3)$ has been designed, with the elementary oscillator being based on the transistor NE3210S01 [Fig. 1(b)]. The oscillator admittance model is extracted from a HB simulation of this oscillator $[6,9]$, isolated from the system, and considering 7 harmonic terms. For this particular free-running oscillator design, the amplitude derivative of the admittance function has the angle $\operatorname{ang}\left(Y_{V}\right)=3^{\circ}$. The discrete transmission line has been implemented with the varactor diode SMV1232. Fig. 2 shows the variation of $\operatorname{ang}\left(Y_{n b}\right)$ with $V_{\text {bias }}$ for different $L$ values. The inductor $L=1 \mathrm{nH}$ has been chosen. The operation points fulfilling (3) and (4) are $V_{\text {bias } 1}=0.77 \mathrm{~V}$, for the stable interval $\left(-90^{\circ}, 90^{\circ}\right)$, and $V_{\text {bias } 2}=7.31 \mathrm{~V}$, for the stable interval $\left(90^{\circ}, 270^{\circ}\right)$. These points, are indicated in the figure.

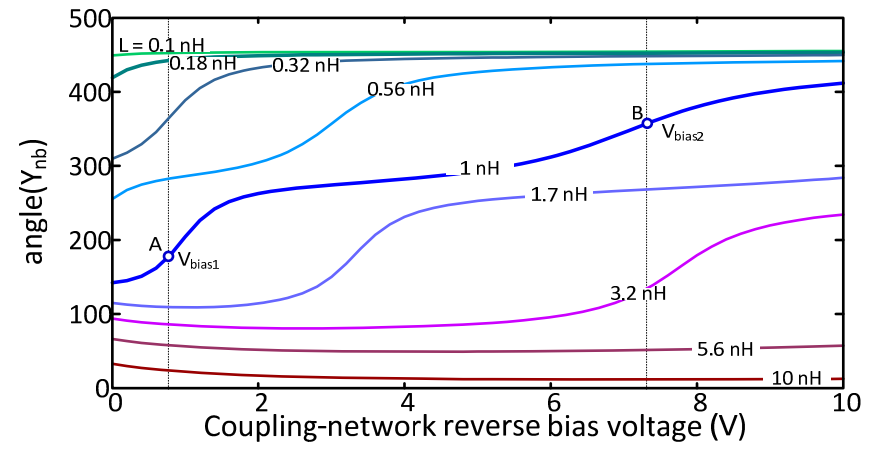

Fig. 2 Inductor-varactor transmission line. Variation of $\operatorname{ang}\left(Y_{n b}\right)$ versus the reverse bias voltage $V_{\text {bias }}$, for different values of the inductance $L$.

For an exhaustive analysis of the system, the matrix $\left[Y_{c}\right]$ in (1), describing the coupling network has been calculated for a whole interval of $V_{\text {bias }}$, going from 0 to $10 \mathrm{~V}$. Note that this analysis versus $V_{\text {bias }}$ is only for exploration purposes, since only the two optimum bias voltages $V_{\text {bias } 1}$ and $V_{\text {bias } 2}$ are necessary. Considering the dependence $\left[Y_{c}\left(V_{\text {bias }}\right)\right]$, there will be an extra analysis parameter $\left(V_{\text {bias }}\right)$, in addition to the tuning voltages of the two outermost oscillators $\Delta \eta_{1}$ and $\Delta \eta_{2}$. Fig. 3(a) presents the variation of the oscillation frequency versus the imposed constant phase shift $\phi$ for different values of $V_{\text {bias }}$, comprising the theoretically optimum design value for the stable phase shift interval $\left(-90^{\circ}, 90^{\circ}\right)$. The stable behavior in this interval will be demonstrated in the next section.

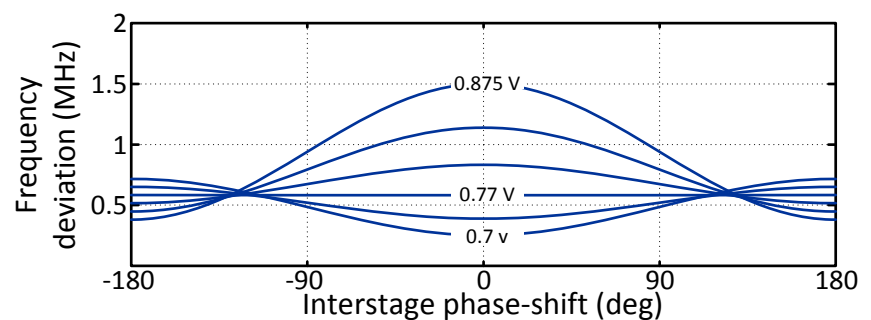

(a)

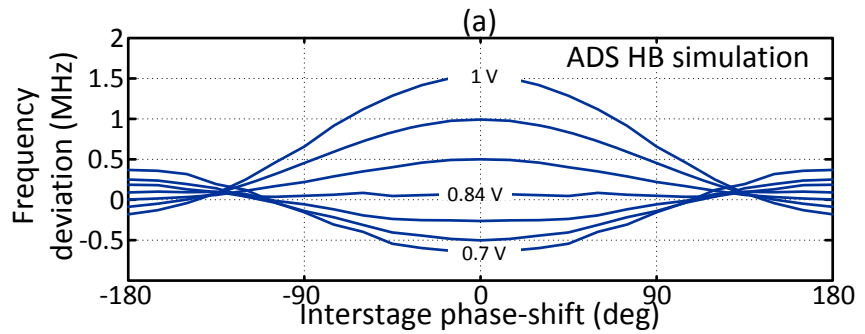

(b)

Fig. 3 Frequency deviation versus the constant inter-stage phase shift for $V_{\text {bias }}$ values about $V_{\text {bias } 1}$. (a) Calculation through (2). (b) Calculation with HB. 
Fig. 3(b) presents a validation with a costly circuit-level simulation of the whole system, using HB with 7 harmonic components. The minimum oscillation frequency deviation is obtained for $V_{\text {bias } 1}=0.77 \mathrm{~V}$ with the semi-analytical formulation and $V_{\text {bias } 1}=0.84 \mathrm{~V}$ with $\mathrm{HB}$. The slight disagreement is attributed to convergence difficulties in the HB circuit level analysis, reflected by the irregular curves, and the fact that the reduced-order model only takes into account coupling effects at the fundamental frequency. Fig. 4(a) and Fig. 4(b) present an analogous study for a $V_{\text {bias }}$ range comprising the theoretically optimum design value for the stable phase shift interval $\left(90^{\circ}, 270^{\circ}\right)$. The stable behavior in this interval will be demonstrated in the next section. The minimum oscillation frequency deviation is obtained for $V_{\text {bias } 2}$ $=7.3 \mathrm{~V}$ with the semi-analytical formulation and $V_{\text {bias } 2}=7.4 \mathrm{~V}$ with HB. Fig. 5(a) and Fig. 5(b) present the variations of the tuning voltages obtained with the semi-analytical formulation and $\mathrm{HB}$ for $V_{\text {bias } 1}$ and $V_{\text {bias } 2}$, respectively. Conditions (3) and (4) give rise to tuning parameter maxima at $\pm 90^{\circ}$, obtained for $V_{\text {bias } 1}$ and $V_{\text {bias2 }}$, as expected in an optimum design [6].
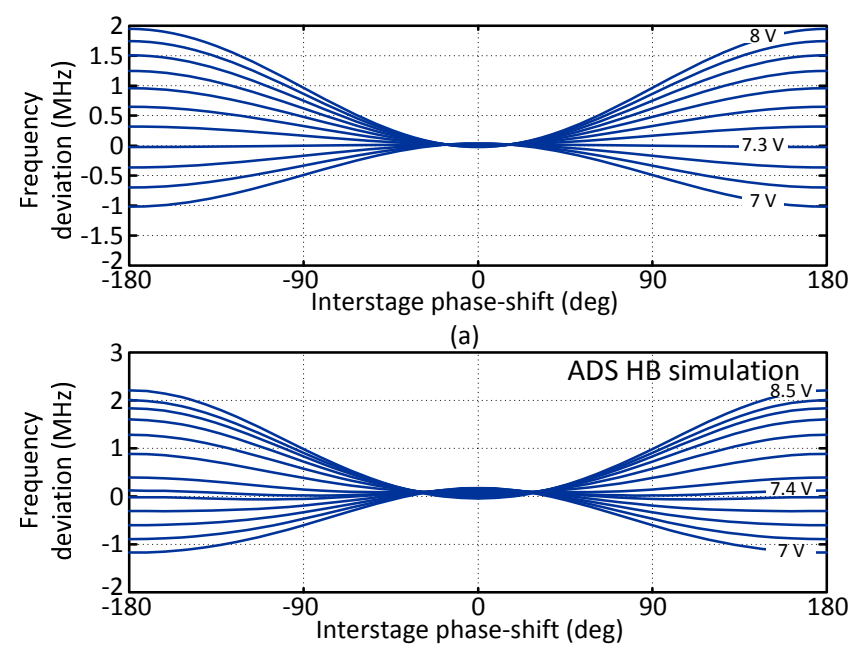

(b)

Fig. 4 Frequency deviation versus the imposed constant inter-stage phase shift for $V_{\text {bias }}$ values about $V_{\text {bias2 }}$. (a) Calculation through (2). (b) Calculation with HB.

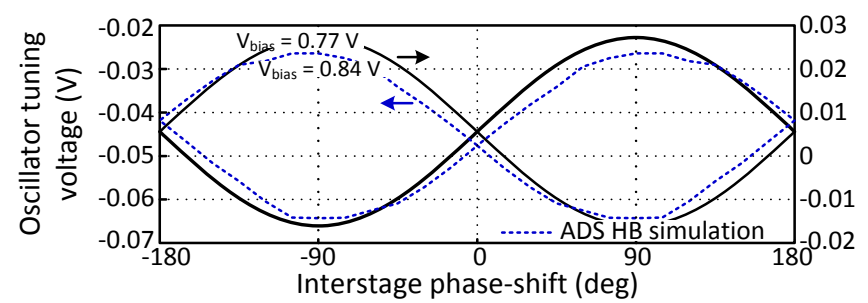

(a)

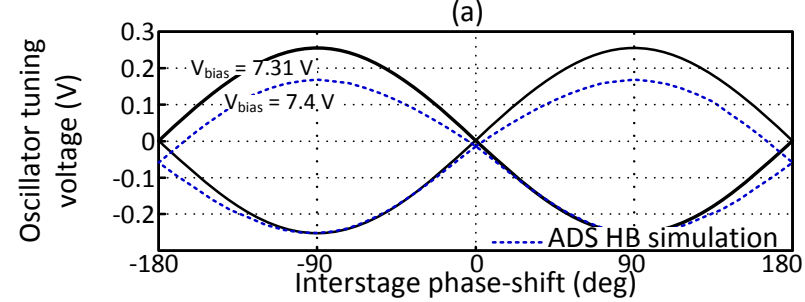

(b)

Fig. 5 Required values of the oscillator tuning voltage for each constant interstage phase shift, obtained with the formulation in (2) and with $\mathrm{HB}$, with 7 harmonic terms. (a) For $V_{\text {bias } 1}$. (b) For $V_{\text {bias } 2}$.

\section{STABILITY ANALYSIS}

Validation of our initial stability assumptions is essential since coupling networks with a topology different from the standard one might alter the expected stability properties. The stability analysis predicts the response of system (2) to small perturbations. For this analysis, small increments are considered in the amplitude, frequency and phase values, taking into account that the product by the frequency increment acts like a time-domain differentiator. Unlike the analyses in previous works, the frequency derivatives of the coupling matrix parameters, $Y_{e \omega}$ and $Y_{n b \omega}$, are taken into account in the formulation, which leads to the linear time invariant system $\Delta \dot{\bar{X}}(t)=M_{\dot{X}}^{-1} M_{X}(\phi) \Delta \bar{X}(t)$, where the vector $\Delta \bar{X}(t)$ is defined as $\Delta \bar{X}=\left(\Delta \bar{V}^{\mathrm{t}} \Delta \bar{\phi}^{\mathrm{t}}\right)$ and the matrixes are given by:

$$
\begin{aligned}
& M_{\dot{X}}=\left[\begin{array}{cccccc}
\frac{Y_{\omega}^{i}+Y_{e \omega}^{i}}{V_{0}} & \frac{Y_{n b \omega}^{i}}{V_{0}} & 0 & Y_{\omega}^{r}+Y_{e \omega}^{r} & Y_{n b \omega}^{r} & 0 \\
-\frac{Y_{\omega}^{r}+Y_{e \omega}^{r}}{V_{0}} & -\frac{Y_{n b \omega}^{r}}{V_{0}} & 0 & Y_{\omega}^{i}+Y_{e \omega}^{i} & Y_{n b \omega}^{i} & 0 \\
\frac{Y_{n b \omega}^{i}}{V_{0}} & \frac{Y_{\omega}^{i}+2 Y_{e \omega}^{i}}{V_{0}} & \frac{Y_{n b \omega}^{i}}{V_{0}} & Y_{n b \omega}^{r} & Y_{\omega}^{r}+2 Y_{e \omega}^{r} & Y_{n b \omega}^{r} \\
-\frac{Y_{n b \omega}^{r}}{V_{0}} & -\frac{Y_{\omega}^{r}+2 Y_{e \omega}^{r}}{V_{0}} & -\frac{Y_{n b \omega}^{r}}{V_{0}} & Y_{n b \omega}^{i} & Y_{\omega}^{i}+2 Y_{e \omega}^{i} & Y_{n b \omega}^{i} \\
0 & \frac{Y_{n b \omega}^{i}}{V_{0}} & \frac{Y_{\omega}^{i}+Y_{e \omega}^{i}}{V_{0}} & 0 & Y_{n b \omega}^{r} & Y_{\omega}^{r}+Y_{e \omega}^{r} \\
0 & -\frac{Y_{n b \omega}^{r}}{V_{0}} & -\frac{Y_{\omega}^{r}+Y_{e \omega}^{r}}{V_{0}} & 0 & Y_{n b \omega}^{i} & Y_{\omega}^{i}+Y_{e \omega}^{i}
\end{array}\right] \\
& M_{X}=-\left[\begin{array}{cccccc}
Y_{v}^{r} & 0 & 0 & a & -a & 0 \\
Y_{v}^{i} & 0 & 0 & b & -b & 0 \\
0 & Y_{v}^{r} & 0 & c & d & -a \\
0 & Y_{v}^{i} & 0 & e & f & -b \\
0 & 0 & Y_{v}^{r} & 0 & c & -c \\
0 & 0 & Y_{v}^{i} & 0 & e & -e
\end{array}\right]
\end{aligned}
$$

In the above matrixes, the subindex indicates the variable with respect to which the derivative is calculated, the superindex indicates real and imaginary part, and:

$$
\begin{aligned}
& a=Y_{n b}^{i} \cos (\phi)+Y_{n b}^{r} \sin (\phi) ; \quad b=-Y_{n b}^{r} \cos (\phi)+Y_{n b}^{i} \sin (\phi) \\
& c=-Y_{n b}^{i} \cos (\phi)+Y_{n b}^{r} \sin (\phi) ; \quad e=Y_{n b}^{r} \cos (\phi)+Y_{n b}^{i} \sin (\phi) \\
& d=2 Y_{n b}^{i} \cos (\phi) ; f=-2 Y_{n b}^{r} \cos (\phi)
\end{aligned}
$$

The stability is determined by the 6 eigenvalues of the time constant matrix $M_{\dot{X}}^{-1} M_{X}(\phi)$. The whole coupled-oscillator system behaves in a free-running regime, so one of the matrix eigenvalues must be $\lambda_{1}=0$. For stable behavior, the other 5 eigenvalues must have negative real part. In Fig. 6(a) the real part of the dominant poles has been traced versus the imposed constant inter-stage phase shift $\phi$ for $V_{\text {bias } 1 \text {, providing an }}$ optimum design in the phase shift interval $\left(-90^{\circ}, 90^{\circ}\right)$, and $V_{\text {bias } 2 \text {, enabling an optimum behavior in the phase shift interval }}$ $\left(90^{\circ}, 270^{\circ}\right)$. As can be seen, the discrete implementation of the transmission line in the coupling networks does not alter the 
size of the stable intervals in any of the two cases, which is due to the low value of the frequency derivatives of the coupling-network parameters $Y_{n b}$ and $Y_{e}$, in comparison with $Y_{\omega}$. The transition from the stable interval $\left(-90^{\circ}, 90^{\circ}\right)$ to the stable interval $\left(90^{\circ}, 270^{\circ}\right)$ is due to the change in the angle of $Y_{n b}$, as shown in (3) and (4). The stability inversion occurs continuously but very quickly versus the parameter $V_{\text {bias }}$. To illustrate this, a stability analysis has been carried out versus $V_{\text {bias }}$, while keeping the oscillator tuning voltages $\left(\Delta \eta_{1}, \Delta \eta_{2}\right)$ at those originally required for $\phi=180^{\circ}$. In Fig. 6(b), the variation of the dominant real pole has been traced versus this parameter. The real pole is on the right-hand side of the

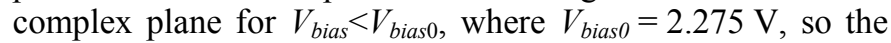
solution is stable for $V_{\text {bias }}>V_{\text {bias } 0 \text {. }}$.

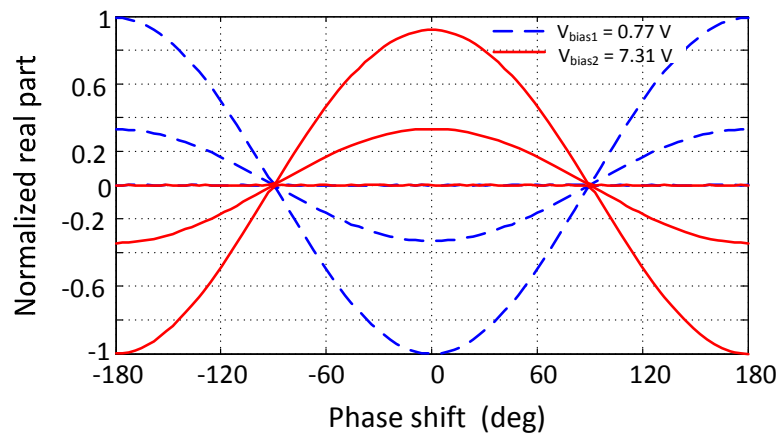

(a)

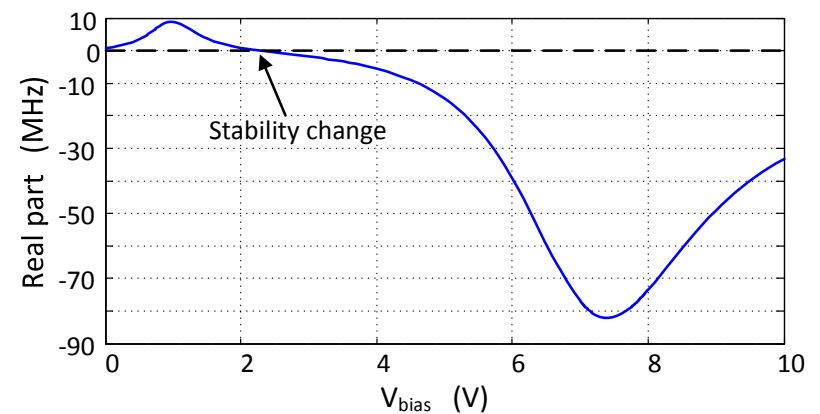

(b)

Fig. 6 Stability analysis with (5). (a) Variation of the real part of the poles versus the imposed constant inter-stage phase shift for $V_{\text {bias } 1}$ and $V_{\text {bias } 2}$. (b) Variation of the dominant real pole of the solution with $\phi=180^{\circ}$ versus $V_{\text {bias }}$. The solution is unstable for $V_{\text {bias }}<V_{\text {bias } 0}$, where $V_{\text {bias } 0}=2.275 \mathrm{~V}$.

The coupled system has been manufactured and characterized experimentally. Fig. 7(a) presents measured waveforms with $\phi=7^{\circ}$, obtained with $V_{\text {bias } 1}$. Fig. $7(\mathrm{~b})$ and Fig.7(c) present measured waveforms with $\phi=150^{\circ}$ and $\phi=175^{\circ}$, obtained with $V_{\text {bias } 2}$. In each case, different pairs of tuning voltages of the outermost oscillators have been used, according to Fig. 5. In the experiment, the solution, originally providing $180^{\circ}$ phase shift, is unstable below $V_{\text {bias } 0}=4 \mathrm{~V}$. The spectrum obtained in the unstable region is shown in Fig. 8.

\section{ACKNOWLEDGMENT}

This work has been supported by the Spanish Government under contract TEC2014-60283-C3-1-R and the Parliament of Cantabria (12.JP02.64069).
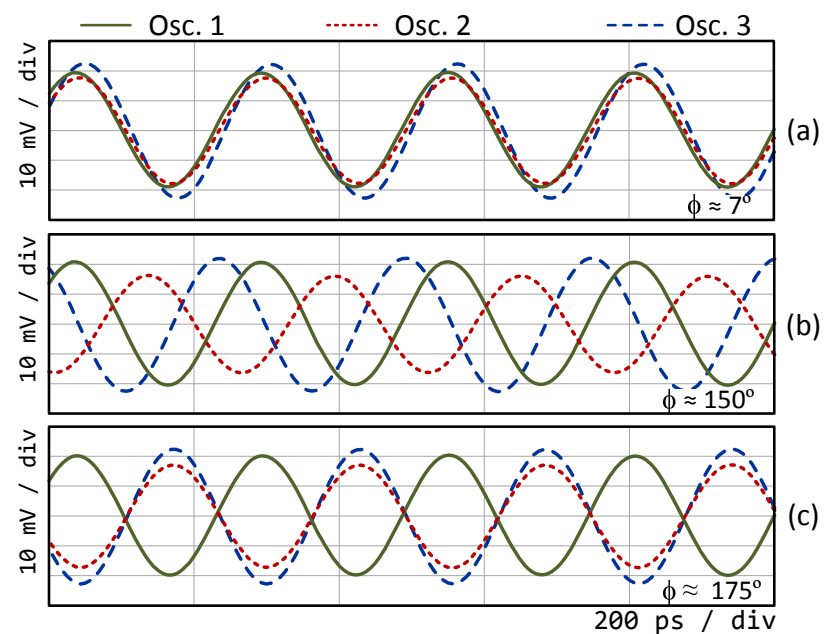

Fig. 7 Experimental waveforms for $V_{\text {bias } 1}$ in (a), and $V_{\text {bias } 2}$, in (b) and (c). In each case, the waveforms are obtained for different pairs of tuning voltages of the outermost oscillators, according to Fig. 5.

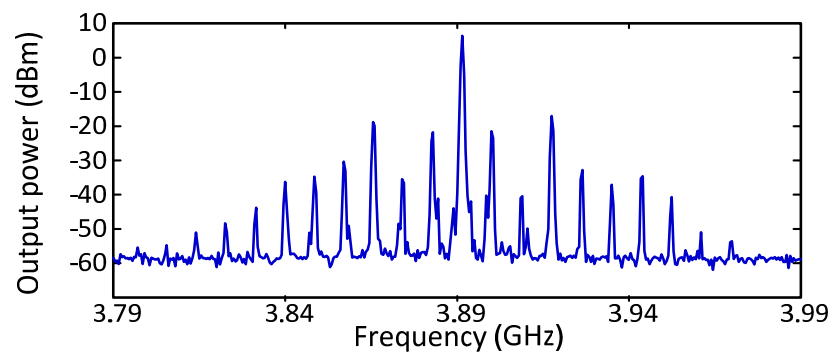

Fig. 8 Experimental spectrum obtained with oscillator tuning voltages corresponding to the solution with $\phi=180^{\circ}$ and $V_{\text {bias }}$ below $4 \mathrm{~V}$.

\section{REFERENCES}

[1] R. A. York, "Nonlinear analysis of phase relationships in quasi-optical oscillator arrays", IEEE Trans. Microw. Theory Techn., vol. 41, no. 10, 1993 pp: 1799 - 1809

[2] P. Liao, R. A. York, "A new phase-shifterless beam-scanning technique using arrays of coupled oscillators", IEEE Trans. Microw. Theory Techn., vol. 41, no. 10, pp. 1810-1815, Oct. 1993.

[3] T. Heath, "Beam steering of nonlinear oscillator arrays through manipulation of coupling phases," IEEE Trans. on Antennas and Propagation, vol. 52, no. 7, pp. 1833-1842, Jul. 2004.

[4] A. Suárez, A. Collado, F. Ramírez, "Harmonic-balance techniques for the design of coupled-oscillator systems in both unforced and injectionlocked operation”, 2005 IEEE MTT-S, Long Beach, Jun., 2005.

[5] F. Ramírez, E. de Cos, A. Suárez, "Nonlinear analysis tools for the optimized design of harmonic-injection frequency dividers", IEEE Trans. Microw. Theory Techn., vol. 51, no. 6, Jun. 2003, pp. 1752-1762.

[6] A. Suárez, F. Ramírez, S. Sancho, "Stability and Noise Analysis of Coupled-Oscillator Systems," IEEE T-MTT, vol. 59, no. 4, pp. 1032 1046, 2011.

[7] H.C. Chang, E. S. Shapiro, R. York, "Influence of the oscillator equivalent circuit on the stable modes of parallel-coupled oscillators, " IEEE Trans. Microw. Theory Techn., vol. 45, no. 8, Aug. 1997, pp. 1232-1239.

[8] M. Rodwell et al. "GaAs Nonlinear transmission lines for picosecond pulse generation and millimetre wave sampling," IEEE Trans. Microw. Theory Tech., vol. 39, no. 7, pp. 1194-2004, 1991.

[9] A. Suárez, S. Sancho, F. Ramírez, "General formulation for the analysis of injection-locked coupled-oscillator systems", IEEE T-MTT, vol. 61, no. 12,2013 . 